\title{
Metal-insulator transition in a multilayer system with a strong magnetic field
}

\author{
X. R. Wang and C. Y. Wong* \\ Physics Department, The Hong Kong University of Science and Technology, Clear Water Bay, Hong Kong, China \\ X. C. Xie \\ Physics Department, Oklahoma State University, Stillwater, Oklahoma 74078
}

(Received 17 November 1998)

\begin{abstract}
We study the Anderson localization in a weakly coupled multilayer system with a strong magnetic field perpendicular to the layers. The phase diagram of 1/3 flux quanta per plaquette is obtained. The phase diagram shows that a three-dimensional quantum Hall effect phase exists for a weak on-site disorder. In the intermediate disorder, the system has insulating and normal metallic phases separated by a mobility edge. At an even larger disorder, all states are localized and the system is an insulator. The critical exponent of the localization length is found to be $\nu=1.57 \pm 0.10$. [S0163-1829(99)51008-4]
\end{abstract}

There has been an increasing interest in understanding the localization problem in three-dimensional (3D) or multilayer disordered electron systems in a strong magnetic field. ${ }^{1-7}$ This is largely due to the discovery of the quantum Hall effect (QHE) in a 3D or a multilayer system, ${ }^{1}$ in particular, with the finding of interesting surface states. According to the scaling theory of localization, ${ }^{8}$ all states in a twodimensional (2D) system are localized if only scalar random potential is present. However, in the presence of a strong perpendicular magnetic field, where the time-reversal symmetry is broken, extended states appear in the centers of disorder-broadened Landau bands and give rise to the integer QHE. On the other hand, a 3D system with a scalar random potential can have a mobility edge that separates extended states from localized ones. It is an interesting question to understand how the transport phase diagram evolves as a system changes from $3 \mathrm{D}$ to $2 \mathrm{D}$ by weakening the interlayer coupling in one direction.

In a multilayer or 3D system with an external magnetic field perpendicular to the layers, each energy band will split into several Landau subbands. Unlike the 2D case, these Landau subbands are overlapped with each other because of the third dimension. There may be two possible scenarios for the phase diagram of extended and localized states in the weak disorder limit. In the case of a strong interlayer coupling, one will expect that there are only two mobility edges separating the extended states from the localized states as shown in Fig. 1(a), where we sketched the density of states for a complete band. The system can undergo a conventional metal-insulator transition if the Fermi energy of the system crosses one of the mobility edges. In the metallic phase, electron transport perpendicular to layers, or parallel to the magnetic field, are through the bulk extended states. In the absence of an interlayer coupling, the system consists of a number of $2 \mathrm{D}$ systems. In a strong perpendicular magnetic field, it is well known that there are two mobility edges for each of the Landau subbands, and QHE is expected. It is natural to expect that a weak coupling can broaden the extended state regime, but the localized-extended-localized phase diagram may not change in a weak disorder limit for each of the Landau subbands as shown in Fig. 1(b). This is a very interesting scenario with three possible phases. The system is an insulator when the Fermi energy is at the bottom of the first Landau subband and in the localized regime. On the other hand, the system is a normal metal when the Fermi energy is inside the extended state region. Finally, the system is a $3 \mathrm{D}$ quantized Hall conductor ${ }^{2,3}$ when the Fermi energy is in the localized regime between two Landau subbands. Changing the Fermi energy, the system will undergo insulator-metal and metal-quantized Hall conductor transitions. In this paper, we study the phase diagram of a weakly coupled multilayer system. We show that the second scenario does indeed occur for a system with weak disorder. For strong disorders, there is a mobility edge separating extended
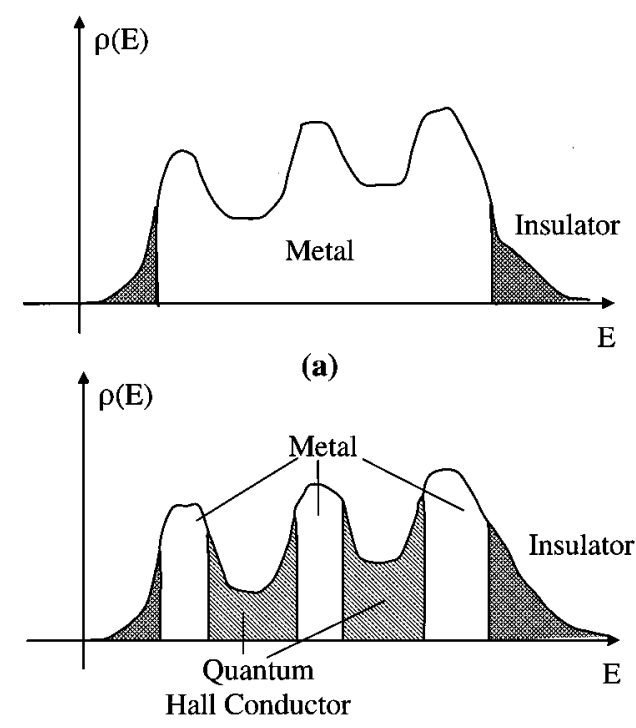

(b)

FIG. 1. Two possible scenarios for a multilayer system in a strong magnetic field perpendicular to the layers. (a) There is only one transition separating localized states from extended states for all Landau subbands. (b) There are mobility edges for each of the Landau subbands, separating localized states from extended states. When the Fermi energy is between two Landau subbands but inside localized states regime, the system is a quantum Hall conductor. 
and localized states, similar to the zero field case.

A natural way to address the issue is to study the Anderson localization model with a nearest-neighbor hopping on a cubic lattice. We assume that an external magnetic field is along the $z$ axis. We consider the case of a strong magnetic field with $1 / 3$ flux quanta per plaquette. The hopping coefficients in the $x$ and the $y$ directions are the same, and their amplitude is chosen as the energy unit. The hopping coefficient along the $z$ direction is one tenth of that in the $x$ or the $y$ direction in order to describe the case of a weak coupling between layers. Therefore, the Hamiltonian of this system can be written as

$$
\mathcal{H}=\sum_{\vec{r}} w_{\vec{r}} c_{\vec{r}}^{+} c_{\vec{r}}+\sum_{\langle\vec{r} ; \vec{\delta}\rangle} t_{\vec{r}, \vec{r}+\vec{\delta}} c_{\vec{r}}^{+} c_{\vec{r}+\vec{\delta}}+\text { c.c. }
$$

where $\vec{r}+\vec{\delta}$ labels the nearest neighbors of site $\vec{r}$. The disorder potential is modeled by the random on-site white-noise potential $w_{\vec{r}}$ ranging from $-W / 2$ to $W / 2$. The hopping coefficients are

$$
t_{\vec{r}, \vec{r}+\vec{\delta}}=\left\{\begin{array}{cc}
e^{\mp 2 \pi \alpha y i}, & \vec{\delta} \in\left\{ \pm \hat{\mathbf{e}}_{x}\right\} \\
1, & \vec{\delta} \in\left\{ \pm \hat{\mathbf{e}}_{y}\right\} \\
0.1, & \vec{\delta} \in\left\{ \pm \hat{\mathbf{e}}_{z}\right\}
\end{array}\right.
$$

describing a system with a uniform magnetic field $B$ in the $z$ direction with the Landau gauge $\vec{A}=(-B y, 0,0)$. The only effect of the magnetic field is on the hopping coefficients through the Peierls phase ${ }^{9} \alpha=2 B / h c$ (we choose lattice constant to be one). Without disorder $(W=0)$, the energy spectrum can be obtained analytically and consists of three bands. The bands are symmetric about the center of the second band, which is zero with the first Landau subband below $E=0$ and the third above $E=0$. In the presence of a disorder, the problem has to be solved numerically. The localization property of the system is still symmetric about $E=0$ even with disorders.

We consider the model on a very long bar geometry of cross section $M \times M$ in the $z x$ plane. The bar is along the $y$ direction. The periodic boundary condition is applied in the $x$ and $z$ directions. Using a standard iteration algorithm, we can calculate the localization length $\lambda_{M}$ at a finite size $M .{ }^{10,11}$ The localization length $\lambda_{M}$ in the critical region is assumed to obey one-parameter scaling law. ${ }^{10,11}$

$$
\Lambda=\frac{\lambda_{M}}{M}=f\left(\frac{\xi(\vartheta)}{M}\right),
$$

where $\xi(\vartheta)$ is the localization length in a 3D system and $\vartheta$ can be either the randomness of on-site energy $W$ or the eigenenergy of the Hamiltonian. $\xi$ can be found by a leastsquares-fitting method with either fixed $E$ or fixed $W$. The localization length diverges near a localized-extended (metal-insulator) transition point as

$$
\xi(\vartheta) \propto\left|\vartheta-\vartheta_{c}\right|^{-\nu} .
$$

The transition points $E_{c}$ or $W_{c}$ can be determined from the localization length $\xi$ from the scaling relation (4). In our numerical calculation, we choose the sample length to be

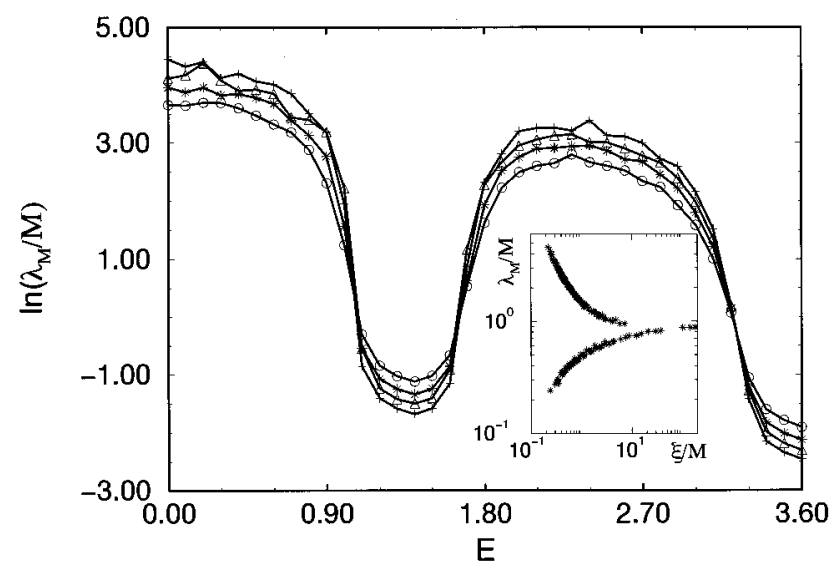

FIG. 2. $\lambda_{M} / M$ as function of energy $E$ with magnitude of random on-site potential $W=2$. For $\bigcirc: M=8 ; *: M=10 ; \triangle: M$ $=12$; and $+: M=14$. Inset: enlargement of the scaling function around the third crossing point $E_{c}=3.22$.

over $10^{5}$ so that the self-averaging effect automatically takes care of the ensemble statistical fluctuations.

We now discuss our numerical results. Figure 2 is the ratio of finite localization length $\lambda_{M}$ to the system width $M$ versus energy $E$ with the strength of disorder $W=2$. The Hamiltonian (1) is symmetric about $E=0$, thus, one needs to plot the curves for $E>0$ only. Different curves are for different system sizes $(\bigcirc: M=8 ; *: M=10 ; \triangle: M=12$; + : $M=14)$. All curves cross at three points of $E \sim 1.06 ; 1.66$; 3.22. Those crossing points indicate that there exist three delocalized-localized transitions. Near the transition region, the normalized localization length $\Lambda=\lambda_{M} / M$ of finite systems of $M=8,10,12,14$ can be described by two nice scaling curves. The inset of Fig. 2 is the two-branch scaling curve near the mobility edge $E_{c}=3.22$. The transition points can also be obtained through curve fitting and we found again that $E_{c}=1.06 ; 1.66 ; 3.22$, in good agreement with the crossing values. The critical exponent for the localization length is found to be about $\nu=1.57 \pm 0.10$, which is consistent with previous results. $^{12-14}$

The results of Fig. 2 show that indeed there is a localized state region, $1.06<E<1.66$, between the second and the third Landau subbands, as far as the transport vertical to the magnetic field direction is concerned. Other approaches, such as the random network approximation, ${ }^{2,3}$ showed that there is a so-called edge-state sheath in this region. The edge-state sheath is extended along the system surface, and electrons can move around through the sheath even at zero temperature. Therefore, the system in the region behaves like a metal in the sense that the conductance of the system along the magnetic field is finite at zero temperature. ${ }^{2,3,5}$ However, because the edge-state sheath exists near the surface of a sample, the conductance along the magnetic field depends on the perimeter of the sample rather than on the area of the sample cross section as a normal metal does., ${ }^{2,3,5}$ Just as the 2D edge states give rise to the quantum Hall effect, this edge-state sheath makes the Hall conductance quantized in 3D. Thus, the system in this region is also called the quantized Hall conductor. ${ }^{2,3}$ The existence of the edge-state sheath may also be understood from that of its $2 \mathrm{D}$ counterpart of edge states. It has been shown that an extended state 


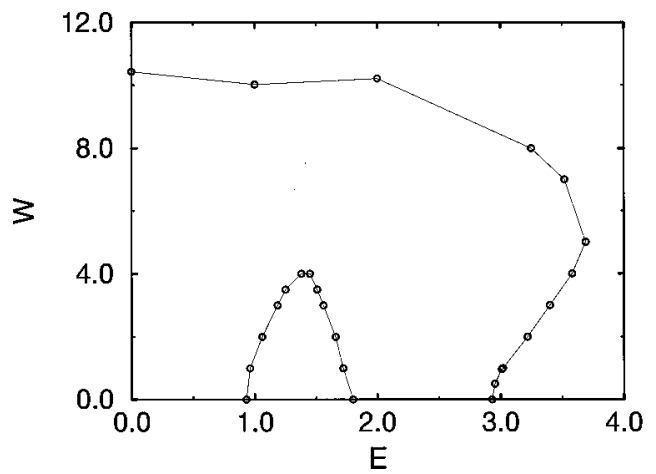

FIG. 3. Phase diagram on the $E_{c}-W$ plane. The states inside the phase boundary are extended while those outside are localized. The systems in the middle pocket are in the quantum Hall conductor phase.

can only be destroyed through scattering involved states in different Landau subbands. ${ }^{15}$ When the disorder is too weak to induce significant mixing among states of different Landau subbands, the extended states are preserved in the form of edge states. The above argument is also consistent with the conservation of the Chern number. ${ }^{15}$

We have carried out numerical calculations for many points on the $W-E_{c}$ plane for the multilayer system. Presented in Fig. 3 is the resulting phase diagram. $E=0$ corresponds to the band center of the middle band, and, again, the localization properties of the first band and the third band are the same (the system is symmetric about $E=0$ ) so we need only to consider $E>0$. There are several interesting features about the phase diagram. (1) At weak disorders $(W<4)$, there are two mobility edges to separate localized and extended states in each of the Landau subbands. Correspondingly, there are three delocalized-localized transition points in the region of $E>0$. The first transition point at small $E$ is one of the two mobility edges for the middle band. The other two are the two mobility edges of the third Landau subband. The first transition point is the one from normal metal to the quantum Hall conductor. The second transition point corresponds to the one from the quantum-Hall-conductor phase to the normal metallic phase. The last one at larger $E$ (band edge) describes the transition from the normal metallic phase

*Present address: School of Physics and Astronomy, University of Minnesota, 116 Church Street SE, Minneapolis, MN 55455.

${ }^{1}$ H. L. Stormer et al., Phys. Rev. Lett. 56, 85 (1986).

${ }^{2}$ J. T. Chalker and A. Dohmen, Phys. Rev. Lett. 75, 4496 (1995).

${ }^{3}$ L. Balents and M. P. A. Fisher, Phys. Rev. Lett. 76, 2782 (1996).

${ }^{4}$ T. Ohtsuki, B. Kramer, and Y. Ono, J. Phys. Soc. Jpn. 62, 224 (1993); M. Hennecke, B. Kramer, and T. Ohtsuki, Europhys. Lett. 27, 389 (1994).

${ }^{5}$ D. P. Druist et al., Phys. Rev. Lett. 80, 365 (1998).

${ }^{6}$ S. Hill et al., Phys. Rev. B 55, R4891 (1997).

${ }^{7}$ R. Fisch, Phys. Rev. B 53, 6862 (1996).

${ }^{8}$ P. A. Lee and T. V. Ramakrishnan, Rev. Mod. Phys. 57, 287 (1985).

${ }^{9}$ R. Peierls, Z. Phys. 80, 763 (1933); J. M. Luttinger, Phys. Rev. to an insulating phase. Thus, at a weak disorder, there is a $3 \mathrm{D}$ quantum Hall regime between the first and the second transition points. This regime decreases with the disorder. Varying the Fermi energy by increasing the electron density, the system can enter consecutively into insulator, normal metal, 3D quantum Hall conductor, and normal metal again. (2) The two inner mobility edges of the phase diagram meet at a point around $W=4$ to close the pocket of the $3 \mathrm{D}$ quantum Hall effect regime. At the intermediate disorder $(4<W$ $<10.5$ ), the disorder causes the three Landau subbands to couple completely together and to form a large energy band. There is only one localized-delocalized transition corresponding to the conventional metal-insulator transition. (3) At strong disorder $(W>10.5)$, all states are localized, and the system can only be in an insulating phase. It is interesting to notice that the disorder makes an electron state more localized, and also broadens the Landau subbands. Below a certain value of disorder, the broadening effect dominates over the localization effect, and the phase boundary shifts into the localized states regime as the disorder increases. Above a certain disorder strength, the localization effect dominates, and the extended state region decreases with disorder, in agreement with previous results. ${ }^{12-14}$

Before we end this paper, we would like to mention a recent study by Drose et al. ${ }^{14}$ who investigated an isotropic Anderson model in a strong magnetic field of $1 / 3$ flux quanta per plaquette. They did not observe the quantum-Hallconductor phase. Their phase diagram contains only the outmost boundary of Fig. 3 of the current work. This is due to the strong layer-layer coupling in their work that mixes different Landau subbands such that the quantum Hall conductor phase cannot exist in the presence of a disorder.

In summary, we have demonstrated that there is a region of 3D quantum Hall effect in a multilayer system with weak interlayer coupling at weak disorders. For large disorders, the physics is similar as for the zero field case, namely, with a mobility edge separating extended and localized states.

We thank Dongzi Liu and Qian Niu for many helpful discussions and comments. X. R. Wang was supported by UGC, Hong Kong, through an RGC/DAG grant. X. C. Xie was supported by the DOE under Contract No. DE-FG0398ER45687.
84, 814 (1951).

${ }^{10}$ W. L. Chan, X. R. Wang, and X. C. Xie, Phys. Rev. B 54, 11213 (1996).

${ }^{11}$ X. C. Xie, X. R. Wang, and D. Z. Liu, Phys. Rev. Lett. 80, 3563 (1998).

${ }^{12}$ A. MacKinnon and B. Kramer, Z. Phys. B-Condensed Matter 53, 1 (1983).

${ }^{13}$ B. R. Bulka, B. Kramer, and A. MacKinnon, Z. Phys. B-Condensed Matter 60, 13 (1985).

${ }^{14}$ T. Drose, M. Batsch, I. K. Zharekechev, and B. Kramer, Phys. Rev. B 57, 37 (1998).

${ }^{15}$ D. Z. Liu, X. C. Xie, and Q. Niu, Phys. Rev. Lett. 76, 975 (1996); X. C. Xie, D. Z. Liu, B. Sundaram, and Q. Niu, Phys. Rev. B 54, 4966 (1996). 